\title{
Las edades de la migración boliviana en Argentina: Córdoba y Ushuaia como destino ${ }^{*}$
}

\author{
The ages of bolivian migration in Argentina: \\ Cordoba and Ushuaia as destination places
}

\author{
María José Magliano** \\ Centro de Investigaciones y Estudios sobre Cultura y Sociedad, \\ Consejo Nacional de Investigaciones Científicas y Técnicas/ \\ Universidad Nacional de Córdoba, Córdoba, Argentina
}

\author{
Ana Inés Mallimaci Barral ${ }^{* * *}$ \\ Instituto Interdisciplinario de Estudios de Género, \\ Universidad de Buenos Aires, Buenos Aires, Argentina
}

Recibido: 25 de marzo de 2014. Aprobado: 28 de mayo de 2015

\begin{abstract}
Resumen
A partir de los resultados de un trabajo de campo cualitativo realizado en dos ciudades argentinas, Córdoba y Ushuaia, el propósito de este trabajo es la comparación socio-histórica de las migraciones de personas nacidas en Bolivia en dichas ciudades de acuerdo al momento de su arribo, las diferentes localizaciones territoriales, las formas que adquiere

Este artículo es el resultado del diálogo de dos investigaciones individuales ("Estrategias y trayectorias migratorias de mujeres y varones bolivianos y peruanos en Córdoba: implicaciones de género, etnicidad y clase social" y "Trabajo, cuidados y migración”, respectivamente), financiadas por el Consejo Nacional de Investigaciones Científicas y Técnicas (CONICET) en el marco de la carrera de Investigador Científico.

* Doctora en Historia por la Universidad Nacional de Córdoba, Argentina. Investigadora Adjunta del Consejo Nacional de Investigaciones Científicas y Técnicas (CONICET) con lugar de trabajo en el Centro de Investigaciones y Estudios sobre Cultura y Sociedad (CIECS-CONICET/UNC). Profesora de la Universidad Nacional de Córdoba en la Facultad de Filosofía y Humanidades. En la actualidad coordina el Programa de Investigación "Multiculturalismo, migraciones y desigualdad en América Latina" (CIECS-CONICET/UNC). Correo electrónico: majomagliano@gmail.com

*** Doctora en Ciencias Sociales por la EHESS de París, Francia en co-tutela con la Universidad Nacional de Buenos Aires, Argentina. Investigadora Asistente del Consejo Nacional de Investigaciones Científicas y Técnicas (CONICET) con lugar de trabajo en el Instituto Interdisciplinario de Estudios de Género. Profesora de la Universidad Nacional de Florencio Varela en el Instituto de Ciencias Sociales en donde participa de líneas de investigación vinculadas al trabajo, género y migración. Correo electrónico: anamallimaci@yahoo.com.ar
\end{abstract}


la migración así como también las principales trayectorias laborales en los lugares de destino. La categoría edades de la migración, acuñada por Sayad (1977), es recuperada aquí para pensar las complejidades y diferenciaciones de la presencia continua e histórica de migrantes bolivianos en el territorio nacional y sus trayectorias que manifiestan distintos momentos de la migración. El análisis que proponemos parte de la premisa que la historia de la propia migración boliviana hacia Argentina se expresa en un saber hacer migratorio acumulado y naturalizado a través de vastas generaciones de migrantes que condiciona las formas y repertorios migratorios disponibles de varones y mujeres, especialmente cuando la migración resulta una estrategia para la reproducción familiar.

Palabras claves: migración boliviana, edades de la migración, trayectorias migratorias.

\begin{abstract}
Starting from a qualitative study developed in two Argentinean cities, Córdoba y Ushuaia, this paper sets out to make a socio-historical comparison between the migration of people born in Bolivia in those cities, taking into account the historical moments of migrants arrival, the diverse territorial localizations within the country, migration forms as well as the main labor trajectories in destination places. The category ages of migration, endorsed by Sayad (1977), is recuperated here to think about the complexities and particularities of the constant and historical presence of Bolivian migrants in the national territory and their trajectories that reveal different moments of migration. This paper discusses the premise that the history of Bolivian migration to Argentina can be expressed as a migration expertise accumulated and naturalized throughout generations of migrants which influences the migratory forms and repertoires available for men and women, especially when migration is a strategy of family reproduction.
\end{abstract}

Keywords: bolivian migration, ages of migration, migration trajectories. 


\section{Introducción}

Las migraciones bolivianas hacia Argentina han sido producto de diversas, complejas e importantes investigaciones en los últimos decenios que la han convertido en el objeto privilegiado de los estudios migratorios en este país. El artículo que presentamos se inscribe en esta tradición con un objetivo que intenta aportar nuevas reflexiones a partir de la reconstrucción en términos comparativos de los rasgos principales de la migración boliviana hacia las ciudades de Córdoba y Ushuaia ${ }^{1}$. La hipótesis que organiza el trabajo supone la importancia de la localización de los fenómenos migratorios, es decir, las características de la migración a nivel nacional adquieren rasgos diferenciales cuando se las analiza desde la realidad concreta de las ciudades cuyas historias, urbanización, poblamiento y especificidades del mercado de trabajo dimensionan de una manera específica las fronteras dentro de las cuales tienen lugar las trayectorias de los/as migrantes. Para la puesta en común de los resultados y su potencial comparación fue necesario resituar las historias migratorias particulares en la gran historia de las migraciones bolivianas hacia Argentina teniendo en cuenta los momentos socio-históricos de las llegadas de los/ as migrantes, las diferentes localizaciones territoriales, las formas que adquiere la migración así como también las principales inserciones laborales en los lugares de destino. La categoría edades de la migración, acuñada por Sayad (1977), vuelve inteligible estos procesos y es retomada aquí para pensar las complejidades y diferenciaciones de la presencia continua e histórica de migrantes bolivianos, varones y mujeres, en el territorio nacional y sus diferentes trayectorias que manifiestan distintos momentos de la migración ${ }^{2}$.

El análisis que proponemos parte de comprender a la historia de la migración boliviana hacia Argentina como la expresión de un saber hacer migratorio (Tarrius, 2000), acumulado y naturalizado a través de vastas generaciones de migrantes que condiciona las formas y repertorios migratorios disponibles de varones y mujeres. Tal como lo han mostrado diferentes estudios (Dandler y Medeiros, 1991; Cortés, 2004; Mallimaci, 2012), venir hacia Argentina forma parte de los recursos disponibles, prácticos y siempre a mano para la reproducción familiar de las familias bolivianas.

\footnotetext{
La ciudad de Córdoba se ubica en el centro del país mientras que la de Ushuaia en el extremo sur.

2 La categoría edades de la migración para Sayad supone el análisis de "las condiciones diferenciales que, en momentos dispares, producen diferentes generaciones de migrantes y diversas trayectorias" (Sayad, 1977, p. 60). En este artículo, al no trabajar en profundidad las condiciones que generan emigrantes, es decir, las variables relativas a las sociedades de origen, no hacemos honor al profundo trabajo sociológico de Sayad. Utilizamos, por tanto, esta categoría para dar cuenta de las especificidades de la larga tradición migratoria boliviana en el país.
} 
La llegada de personas bolivianas hacia Córdoba y Ushuaia ocurre en momentos diferenciales de la historia de la migración boliviana en el país, compartiendo rasgos comunes a estas edades de la migración y otros que le son propios. La caracterización de las edades se realizará en base a trabajos previos como el de Benencia (2012), que describe el proceso histórico que posibilitó el avance de las familias bolivianas sobre el eslabón comercial de la cadena agroalimentaria de la producción hortícola en fresco a partir de la constitución de enclaves étnicos en distintos territorios de Argentina; el de Benencia y Karasik (1995), que reconstruye la historia de la presencia boliviana en el país desde principios del siglo XX; el de Sassone (2009), que establece una serie de estadios (migración fronteriza, regional y transnacional) de este proceso a partir de retomar una geografía histórica de la migración; el de Zalles (2002), que plantea una periodización de acuerdo a la legitimación integral del migrante como sujeto económico y social ${ }^{3}$. Los resultados de nuestra indagación se ubicarán en diferentes edades de la migración no sólo por ocurrir en diferentes momentos históricos sino porque resultan expresivos de procesos sociales y económicos de generación de migrantes diferenciales.

En términos metodológicos, este artículo se asienta en diferentes estrategias y técnicas de recolección de datos. Para la reconstrucción de la primera edad de la migración nos valdremos de datos secundarios provenientes de las investigaciones ya desarrolladas sobre la migración boliviana hacia Argentina en este período histórico. Para la reconstrucción de los rasgos principales de la migración boliviana hacia las ciudades de Córdoba y Ushuaia (lo que incluimos en la segunda y tercera edad de la migración boliviana en el país), nos apoyamos en técnicas cualitativas de análisis, en especial observación participante y entrevistas en profundidad realizadas a varones y mujeres bolivianos/as tanto en Córdoba como en Ushuaia con el fin de indagar sobre sus trayectorias migratorias, laborales y representaciones sociales sobre las construcciones comunitarias en las ciudades de residencia ${ }^{4}$.

El artículo contará así de tres secciones principales: primero, la reconstrucción de las edades de la migración boliviana hacia Argentina a partir de la literatura disponible

3 El campo de los estudios sobre migración boliviana en Argentina ha sido muy prolífico desde las últimas décadas del siglo pasado en adelante. En tal sentido, la selección de textos realizada para este artículo no intenta ser exhaustiva ni reconstruir el completo estado de la cuestión de este campo de estudios.

4 En la ciudad de Córdoba se entrevistaron a 30 personas nacidas en Bolivia. Son 20 mujeres y 10 varones nacidos/as en Cochabamba (15), Tarija (5), Potosí (5) y el resto de los casos en La Paz, Oruro y Sucre. En la ciudad de Ushuaia se entrevistaron a 45 personas nacidas en Bolivia. Son 30 mujeres y 15 varones nacidas/ os en Cochabamba (19), Potosí (14), y el resto de los casos en Oruro, La Paz, Sucre y Tarija. Asimismo, en ambos lugares se llevó a cabo observación participante con el fin de reconstruir los contextos cotidianos y extracotidianos de la sociabilidad boliviana tanto en Córdoba como en Ushuaia.

144 Si Somos Americanos. Revista de Estudios Transfronterizos 
que permitirá enmarcar el análisis propuesto; segundo, la caracterización de la migración hacia la ciudad de Córdoba; tercero, la caracterización de la migración hacia la ciudad de Ushuaia. Las dimensiones que serán recuperadas para identificar las especificidades de cada momento y trayectoria migratoria en los distintos marcos espaciales son: el período de llegada de personas bolivianas, su vinculación con procesos demográficos, económicos y sociales más generales, los modos de migrar, las formas y estrategias de vinculación con el territorio y el espacio urbano, y las inserciones laborales de estos migrantes en los lugares de destino.

\section{Las edades de la migración boliviana en Argentina}

Los desplazamientos de personas nacidas en los países vecinos hacia Argentina tienen una larga historia. Balan (1985) ha definido la existencia de un "sistema migratorio del Cono Sur" en el cual tradicionalmente Argentina ha sido un país de destino. En particular, la migración boliviana puede visibilizarse en las estadísticas desde los inicios del Estado nacional en la segunda mitad del siglo XIX ${ }^{5}$. Desde ese momento y hasta la actualidad, ha habido un proceso constante y estable en el stock cuantificable de migrantes limítrofes, representando entre el $2 \%$ y $3 \%$ del total de la población argentina (Instituto Nacional de Estadística y Censos, 2012, p. 91).

Las migraciones bolivianas hacia Argentina se han constituido históricamente como una estrategia familiar de reproducción social ${ }^{6}$ antigua y extendida, siendo procesos de largo plazo, donde pasado, presente y futuro se interrelacionan (Mallimaci, 2012; Hinojosa, 2009). Primero se concentraron en las zonas agrícolas fronterizas del norte del país (Jujuy y Salta principalmente) y luego, de manera progresiva desde mediados del siglo XX, los/as migrantes bolivianos/as se dedicaron, por un lado, a la cosecha de la vid en Mendoza, a la recolección de manzanas y peras en Río Negro y, por otro, acompañaron el proceso de crecimiento del área de influencia de la ciudad de Buenos Aires y otras zonas urbanas del país, como Córdoba, debido a la concentración de industrias manufactureras

5 Para la oficina de estadísticas nacionales (INDEC) la definición de inmigrante se computa según el país de nacimiento.

6 El concepto de estrategia familiar de reproducción social apunta a las actividades relacionadas con el mantenimiento y reproducción del grupo familiar. Su uso desplaza la comprensión sobre las causas migratorias del individuo al grupo familiar comprendido como un conjunto asimétrico y conflictivo de relaciones sociales. En esta investigación se utiliza desde la perspectiva de Bourdieu que la comprende como conjunto de prácticas que tienen una dirección o intencionalidad objetiva, sin ser conscientemente asumida, de reproducir una fracción de la sociedad, es decir, la conservación o la mejora de sus condiciones de vida (Bourdieu, 2011). 
y de servicios que resultaron polos de atracción para esta población (Benencia, 2003, p. 424). Paralelamente a estos procesos, la migración europea -preponderante desde el siglo XIX hasta mediados del siglo XX- disminuye de tal modo que según datos del último censo el 77\% de la población extranjera proviene de países limítrofes y del Perú. De este modo, estudiar el fenómeno de la migración en el contexto de Argentina reciente implica necesariamente referirse a la migración limítrofe.

En Bolivia, por su parte, las migraciones han sido un fenómeno social central y persistente en la historia de ese país. Los procesos migratorios, internos y externos, vienen desarrollándose con diversa intensidad desde el siglo XIX en adelante siendo estructurales y constitutivos del devenir histórico boliviano (Cortés, 2004, p. 168). Para muchas familias, la migración -interna y externa- ha resultado una opción dentro de un conjunto de distintas estrategias familiares y de subsistencia (Dandler y Medeiros, 1991, p. 21).

De modo semejante a lo registrado en otras investigaciones (Bendini y Radonich, 1999; Benencia, 1997; Bologna, 2007; Domenach y Celton, 1998; Hinojosa, 2009; Pizarro y Trpin 2011; Rivero, 2012; Sassone, 1988) una parte importante de los/as entrevistados/ as, tanto en Córdoba como en Ushuaia, experimentaron movimientos previos a la migración hacia Argentina, especialmente quienes provienen de sectores rurales. Se trata de fenómenos que junto con la pluralidad de destinos migratorios se acumulan como experiencias pasadas de movilidad. Esas experiencias forman parte de un bagaje personal y colectivo adherido al cuerpo y al conocimiento de las personas, que las sitúa en una relación con el espacio y la permanencia diferente a aquella experimentada por quienes viven la sedentariedad como norma y que en algún momento deben migrar. Para muchos/as, el movimiento migratorio no es vivido como un momento excepcional en un contexto vital de asentamientos residenciales sino que, por el contrario, es la misma cotidianeidad la que se define como móvil territorialmente. En términos generales, no existe por lo tanto una migración que quiebre las experiencias, sino movimientos de diferentes grados de importancia que se superponen a lo largo del trayecto. Las migraciones y/o movilidades son experimentadas más como un modo de vida que como un momento singular de estas vidas (Pries, 2002).

7 Las dinámicas migratorias hacia Argentina, España y Estados Unidos ocupan un lugar relevante dentro del campo de los estudios sobre migraciones internacionales en Bolivia, como lo expresan las investigaciones de Baby-Collin y Cortés (2014), Hinojosa (2004 y 2009), De la Torre Ávila (2006), Farah (2005), Ferrufino (2007), entre otras. 


\section{La primera edad de la migración boliviana en Argentina: el norte como destino ${ }^{8}$}

Tal como lo hiciera Sassone (1988), es posible identificar este momento histórico del proceso migratorio boliviano hacia Argentina, que abarca el siglo XIX y mediados del siglo XX, como migración fronteriza $a^{9}$. En el transcurso de este período, el flujo migratorio boliviano se concentra principalmente en las zonas de la frontera norte, dedicándose especialmente a tareas rurales, trabajos zafrales y estacionales (siempre como peones rurales, el escalafón más bajo del trabajo rural). La migración boliviana expresa una población de frontera, con idas y venidas constantes entre país de origen y destino. Sin embargo, estas circulaciones son diferentes a las de los típicos trabajadores temporarios que se trasladan en épocas de cosecha y vuelven a sus hogares una vez terminada la actividad para repetir la movilidad cuando la cosecha lo requiere, dado que los/as bolivianos/as suelen encadenar diferentes actividades, no sólo cosechas entre sí sino también cosechas con otros trabajos temporarios (Reboratti, 1983). Esto impacta en las formas de migración, no ya pendulares sino que son las estadías (definidas como temporales) las que se conforman como destinos múltiples encadenados.

Esta primera edad de la migración boliviana hacia Argentina se caracteriza por su concentración en la región del norte argentino ${ }^{10}$, debido tanto a la cercanía geográfica como a la importancia de las características de los mercados de trabajo en los que se insertaban: la cosecha de la caña de azúcar -la zafra- que implicaba la modalidad de trabajo por temporadas. Esta actividad suponía una demanda de mano de obra (masculina y femenina) muy localizada en el tiempo, ya que las tareas se completaban entre mayo y septiembre, práctica que resultaba adecuada a las necesidades de trabajo de algunos migrantes que poseían una producción familiar en Bolivia (Bologna, 2007).

A partir de la década del 60, en un contexto de crisis de las economías regionales y de pérdida de posiciones relativas de la población boliviana en los mercados de trabajo de las zonas fronterizas (Marshall y Orlansky, 1983, p. 45), un amplio conjunto de los y las bolivianos/as comienza a dirigirse hacia las principales ciudades argentinas. Si bien

8 De las diferentes edades que aquí se presentan, esta es la única que no podemos trabajar desde los datos empíricos construidos en nuestras propias investigaciones por lo que nos basaremos en datos secundarios.

9 La frontera es concebida aquí como un espacio de constante interacción, constitutivo de redes y cadenas que superan las lógicas de Estado y la división nacional - extranjero.

10 Para el caso de Jujuy, y a diferencia de otros distritos del país donde la presencia boliviana se recorta contra perfiles demográficos, sociales y patrones socio-culturales y hasta fenotípicos contrastantes, los/as bolivianos/as han configurado históricamente una categoría no siempre diferenciable del conjunto de las poblaciones locales (Karasik, 2011, p. 401). 
la migración hacia las ciudades no se inicia aquí, en el marco del argumento sostenido en este trabajo resulta significativa la transformación de las condiciones estructurales de la migración boliviana en Argentina, dando inicio a la segunda edad de la migración, en la cual se sitúa la ciudad de Córdoba. El norte deja de ser el destino único a tal punto que la lectura cuantitativa del stock de población nacida en Bolivia en el censo de 1980 muestra que su presencia en el Área Metropolitana de Buenos Aires supera a la del norte argentino (Balan, 1990).

\section{La segunda edad de la migración boliviana en Argentina: Córdoba en el horizonte de los/as bolivianos/as}

Desde 1960, las migraciones fronterizas de carácter rural-rural fueron paulatinamente suplantadas por migraciones de carácter rural-urbano hacia las grandes ciudades (Buenos Aires, Mendoza y Córdoba especialmente), y reorientadas de forma creciente para el caso de los varones hacia el sector de la construcción (Benencia, 2003). Este cambio obedeció a diversas transformaciones socio-económicas que se sucedieron en Argentina y que pueden encontrarse entre las causas de la migración interna (de nacionales y extranjeros/as) hacia las grandes urbes registradas para esta misma época. Es decir, no se trata de una especificidad de la migración boliviana sino que se relaciona con las condiciones favorables generadas por las políticas de industrialización de los años 30 y 40 en el litoral pampeano como consecuencia de las transformaciones en el modelo socio-económico ${ }^{11}$. Para el caso específico de la migración boliviana, impacta además la crisis en las economías regionales (la caída de los precios del azúcar y la mecanización de los trabajos de recolección de la caña en los ingenios azucareros, la crisis tabacalera en Corrientes o el crack algodonero en Chaco) cuyo efecto es una movilización de la población trabajadora hacia las zonas urbanas, dedicándose mayoritariamente a actividades relacionadas con el sector de los servicios, la construcción y las labores agrícolas en los cinturones verdes de las ciudades (Benencia, 2003; Benencia y Karasik, 1995; Sassone, 1988). Por otra parte, se producen transformaciones importantes en la propia Bolivia que tienen su correlato en los procesos migratorios hacia Argentina. En este sentido, es preciso considerar las impli-

11 Estas políticas de industrialización se basan en la sustitución de importaciones como modelo de acumulación económica, que implicó la transformación de una economía basada primordialmente en un desarrollo hacia fuera para convertirse, en parte, en un desarrollo hacia adentro. Este modelo se mantuvo, no de manera uniforme, hasta la década del 70 y especialmente hasta los años 90, cuando se aplicaron medidas de corte neoliberal que supusieron, entre otros cambios, una apertura externa, la liberalización de los mercados y la reprimarización de la economía. 
cancias de la reforma agraria en Bolivia de 1953 que libera a una proporción importante de la población rural (al eliminar el servicio obligatorio en las haciendas) y que sienta las condiciones para hacer más expedita su migración al extranjero (Zalles, 2002).

Esta edad de la migración boliviana, que se caracteriza principalmente por su orientación hacia las principales ciudades, sobre todo Buenos Aires, transforma las formas corrientes en relación con el territorio expresando proyectos más sedentarios. Sin embargo, esto no significa que la movilidad territorial de la migración boliviana se reduce sino que se modifican sus formas: los circuitos migratorios se organizan en base a una ciudad argentina (y ya no necesariamente el hogar en Bolivia), produciendo en muchos casos una migración circular (Dandler y Medeiros, 1991). A partir de la llegada de migrantes bolivianos a la ciudad de Córdoba, intentaremos reconstruir las especificidades de este momento histórico de la migración boliviana, considerando para ello la localización territorial, el tipo de movilidad y las principales inserciones laborales de los/as migrantes. Si bien la presencia de personas oriundas de Bolivia en Córdoba puede rastrearse hacia comienzos del siglo XX (e incluso antes), es hacia mediados de ese siglo cuando este proceso adquiere mayor dinamismo bajo dos itinerarios principales. Por un lado, una migración de estudiantes de sectores medios y medios altos, en especial varones, quienes llegaron a Córdoba desde las ciudades bolivianas (La Paz, Cochabamba, Sucre y Tarija principalmente) con el propósito de ingresar a la Universidad Nacional de Córdoba ${ }^{12}$. Muchos de estos estudiantes permanecieron en Córdoba una vez concluyeron -o no- sus estudios mientras que otros volvieron a sus lugares de origen. Por el otro, cobra mayor fuerza una migración laboral de carácter familiar, proveniente tanto de zonas urbanas como rurales, orientada de manera principal por el trabajo del varón, en especial en el rubro de la construcción. Según se evidencia en las propias narrativas de los migrantes y de sus familias, esta actividad se ha tornado orientadora del proceso migratorio hacia la ciudad. Rodolfo, un migrante boliviano que llega a Córdoba en 1955 comentaba al respecto ${ }^{13}$ :

En la construcción la mano de obra boliviana es muy calificada y muy solicitada, especialmente en todo lo que se refiere a las molduras, al trabajo sobre yeso, y aparte de eso, la particularidad que tiene un trabajador boliviano es que es completo (Comunicación personal. Córdoba, 2005).

Esto mismo era planteado por Luciano:

12 La Universidad Nacional de Córdoba es la más antigua de Argentina (1613) y posee una larga tradición de formación académica en Argentina y América Latina.

13 Los nombres de las personas mencionadas en este texto han sido modificados para preservar su anonimato. 
En Córdoba todos los diques que se construyeron en los cuarenta, cincuenta, sesenta, tienen mano de obra boliviana, el dique Los Molinos, el San Roque, Cruz del Eje, todos, porque el boliviano tiene una condición especial que no tiene mal de altura, no tiene vértigo, entonces puede andar en los andamios sin problemas, tenían un desplazamiento perfecto sin riesgos laborales. Por otro lado, acá en Córdoba todos los grandes edificios han sido construidos por bolivianos, el caso Torre Ángela por ejemplo [uno de los edificios más tradicionales de la ciudad de Córdoba], allí más del cincuenta por ciento fue mano de obra boliviana. Después acá, todo lo que es el área peatonal también tiene mano de obra boliviana (Comunicación personal, Córdoba, 2007).

La demanda de trabajadores/as bolivianos/as en Córdoba en esas décadas se vincula al propio desarrollo socio-económico de la provincia. La radicación de las fábricas automotrices en Córdoba, como la Kaiser y la Fiat, resultó un motor central para el impulso industrializador de la provincia (Domenach y Celton, 1998). El establecimiento de estas industrias en la ciudad y la provincia, como las militares, las de aviones y las de transporte, entre otras, generaron un significativo desarrollo en esta región. Este proceso, a su vez, repercutió en otras áreas de la economía, incrementándose la demanda para ciertas actividades, sobre todo en las ciudades. De modo que con el arribo de familias migrantes bolivianas a las grandes ciudades surgieron otras inserciones laborales en comparación con aquellas de las provincias del norte del país.

Si durante la primera edad eran los varones o las familias quienes migraban contratadas para las cosechas, los cambios en las trayectorias de este segundo momento histórico impactan en las formas de las migraciones de varones y mujeres. Esas migraciones se asientan en dos dinámicas principales: la primera, quizás la más antigua, es la migración pionera del varón y la relocalización posterior del resto de la familia; la segunda, la migración de la familia en su conjunto. En ambas dinámicas de las migraciones hacia Córdoba, la migración de la mujer ha implicado la migración de los hijos (Magliano, $2013)^{14}$. Salvo para aquellos migrantes que vinieron a la ciudad a estudiar, donde predomina una migración más individual y masculina, la elección de la ciudad de Córdoba se vincula principalmente con la existencia de algún familiar viviendo en ella y el desplazamiento se encuentra motorizado por la búsqueda de oportunidades de inserción laboral del varón pero también de la mujer, quien se orientó a sectores específicos del mercado

14 Esto no implica desconocer que en las migraciones circulares de la primera mitad del siglo XX muchos varones bolivianos se movilizaban con sus hijos. 
laboral cordobés en este período: el pequeño comercio y el trabajo doméstico remunerado principalmente ${ }^{15}$.

Para un importante conjunto de estos migrantes, Córdoba no era el primer destino dentro de Argentina sino que la migración formaba parte de un circuito más amplio que incluía otras regiones del país (Domenech y Celton, 1998). Tal es el caso de Amalia y su familia, aunque con una particularidad, ella nace en Argentina durante el período en que sus padres venían para trabajar en las cosechas en el norte. Sin embargo, siendo un bebé vuelve a Bolivia con sus padres:

E: ¿Cómo llegó a Córdoba?

A: Soy nacida en Argentina, pero mis padres me criaron en Bolivia. Ellos venían acá a trabajar en las cosechas. No acá a Córdoba sino más al norte.

E: ¿Los dos vinieron a trabajar, su mamá y su papá?

A: $\mathrm{Si}$, los dos, mi madre colaboraba mucho en la economía del hogar. Como te decía yo tendría 13 años cuando me volví a la Argentina, fue en el 77 después que mi mamá falleció (...) Somos de un pueblito cerca de Oruro, de ahí son mis padres. Decidí venirme con una prima que me entusiasmó mucho, que era lindo, yo no me acordaba porque era muy bebé cuando me llevaron de vuelta mis padres (...)

E: Entonces, ¿primero llega a Jujuy?

A: Si porque ella estaba en Jujuy, yo trabajaba como doméstica y estudiaba. Ahí (en Jujuy) estuvimos dos años más o menos, y un hermano de mi prima se viene a Córdoba a estudiar porque era sastre en Bolivia, pero a él le gustaba estudiar abogacía también. Se viene para acá y mi prima no aguanta, me dice 'mi hermano está en Córdoba, vamos, vamos. Y nos vinimos, me acuerdo, un 1 de mayo, para el día del trabajador llegamos a Córdoba (...) Hasta que damos con una familia buena y empezamos a trabajar. Y ahí yo hice mis trámites para quedarme, pasó un tiempo, falleció mi abuela, que era lo único que tenía allá que se preocupaba por nosotros y ya no quise volver (Comunicación personal, Córdoba, 2007).

15 En este artículo, concebimos al trabajo de manera amplia, involucrando también su dimensión reproductiva. De acuerdo a lo que hemos reconstruido en nuestro trabajo de campo, para una mujer boliviana, trabajar en el ámbito productivo no implica el desarrollo único de actividades extra domésticas, sino que esas actividades se construyen en conjunto con las tareas domésticas. 
La propia historia de Amalia expresa los sentidos de aquellas idas y venidas de varones y mujeres que mencionábamos como parte de las estrategias familiares de reproducción social. Asimismo, revela que Córdoba no necesariamente representaba el primer destino dentro de Argentina, cuestión que se va a ir modificando en los últimos años del siglo XX. Los/as migrantes que arribaban en los años 60 y 70 explican en parte la elección de Córdoba a partir de ciertas imágenes construidas sobre la ciudad, tal como el prestigio de su universidad o la creciente demanda de trabajo que la convertía en una tierra de posibilidades. A su vez, en el transcurso de estas décadas se fueron consolidando las redes de contacto e información entre familias y vecinos, desempeñando un papel de importancia en la retroalimentación de este proceso migratorio ${ }^{16}$.

En las llegadas a la ciudad, los /as migrantes tendieron a concentrarse en espacios específicos de la ciudad de Córdoba, sobre todo en Villa El Libertador, barrio ubicado en la zona sur de la ciudad que existe como tal desde la década del 30 del siglo XX y que para mediados de siglo pasado estaba prácticamente despoblado. De acuerdo a diversos estudios, en este barrio de la zona sur de la ciudad se ubica la más antigua población boliviana en Córdoba (Bompadre, 2005; Domenach y Celton, 1998; López, 1999). Las experiencias cotidianas de los migrantes bolivianos residentes en Villa El Libertador, según sostiene Bompadre (2005, p. 203), estuvieron atravesadas por diferentes elementos: el lugar de origen, los lazos de parentesco y la existencia de un espacio común que opera como referencia tanto para el que viene como para el que regresa a su tierra y orienta a quienes quieren migrar. Ahora bien, aunque este espacio ha sido históricamente el principal barrio de asentamiento de la población en Córdoba desde la segunda mitad del siglo XX, a partir de las últimas décadas se diversificaron los lugares de destino dentro de la ciudad, incorporándose otros espacios como Pueyrredón, Villa Bustos, Villa Esquieú, El Quebarachal y Nuestro Hogar 3, entre otros, todos ellos caracterizados por ubicarse en la periferia de la ciudad y por su precariedad y marginalidad. Este desplazamiento al interior de la ciudad se vincula principalmente con las transformaciones en la estructura social y económica en el marco del contexto neoliberal y su impacto en los sectores populares (dentro de los cuales se ubican gran parte de la población boliviana en el país).

16 La noción de redes para analizar los procesos migratorios ha sido ampliamente desarrollada dentro de este campo de estudios. En este artículo, la categoría de redes es utilizada para dar cuenta de que los/as migrantes usan las relaciones sociales para constituir sus desplazamientos en el marco de restricciones impuestas por el mercado y los propios conflictos internos de quienes las transitan. Consideramos que los recursos disponibles en las redes no son distribuidos necesariamente de modo igualitario y que la acumulación de contactos e información estructura posiciones dentro de las redes generando relaciones de poder asimétricas. Sin embargo, dado los objetivos específicos de este artículo no podrán trabajarse las desigualdades que las redes estructuran y reproducen sino que son mencionadas como estructuradoras de las llegadas a las ciudades. 


\section{La migración boliviana desde los años 80: el impacto neoliberal}

Tanto en Bolivia como en Argentina, la implementación de políticas de ajuste estructural en los años 80 y 90 para dar respuestas a las crisis hiperinflacionarias y de la deuda externa, repercutieron en las migraciones internacionales. En Bolivia, los efectos sociales y económicos de las políticas neoliberales a partir de 1985 le imprimen nuevos sentidos a las dinámicas migratorias internas como externas, convirtiéndose en una estrategia de supervivencia para cada vez más familias bolivianas ${ }^{17}$. Argentina, asimismo, registró a partir de la década del 90 la vigencia de un régimen cambiario que equiparaba el peso argentino al dólar estadounidense lo cual resultaba atractivo a los/as migrantes en tanto les permitía ahorrar en dólares y enviar remesas a sus lugares de origen (Ceriani et al., 2009, p. 148) $)^{18}$.

A diferencia de la migración boliviana que había arribado a Córdoba -y a otras ciudades de Argentina- hacia mediados del siglo XX, desde la década del 80 la migración boliviana manifiesta otros itinerarios desplegados. De acuerdo a los testimonios de migrantes que hemos podido reconstruir, desde finales del siglo XX hasta la actualidad, Córdoba se convierte en primer destino dentro de Argentina. Cada vez más, las llegadas a la ciudad no venían precedidas de una estancia previa en otra ciudad/región de Argentina sino que lo hacían directamente desde Bolivia, lo que no simboliza que necesariamente permanecieran definitivamente en dicha ciudad.

Asimismo, y bajo el escenario neoliberal, los/as migrantes muestran una mayor diversificación en sus actividades laborales. Si bien la construcción, el pequeño comercio y el trabajo doméstico remunerado continúan siendo nichos laborales importantes para esta población, surgen otros, como la producción hortícola en fresco en el cinturón ver-

17 Si bien históricamente la sociedad boliviana se ha caracterizado por la existencia de profundas asimetrías sociales; en 1985 el gobierno del Movimiento Nacional Revolucionario (MNR) implementa un plan de ajuste estructural, adhiriendo a las principales recetas neoliberales. Entre las medidas comprendidas en ese decreto, se privatizaron las minas -actividad central e histórica de la economía boliviana- generando un éxodo masivo de trabajadores mineros y de quienes subsistían gracias a la vida minera hacia otras zonas del país y también hacia el exterior.

18 Este régimen cambiario finaliza a comienzos del 2002 como corolario de una profunda crisis socio-económica que transitó el país producto de los efectos de las políticas neoliberales. Si bien no nos detendremos aquí en los efectos sociales y económicos del neoliberalismo, es importante destacar, siguiendo a Benencia (1997, p. 64), que durante las décadas del 80 y 90 (en un contexto de crisis económica tanto en Argentina como en Bolivia) muchos migrantes bolivianos insertos en los sectores agrícolas de los grandes centros urbanos experimentan una movilidad social ascendente. 
de, la fabricación de ladrillos y, más recientemente, las tareas textiles ${ }^{19}$. La concentración de personas de origen boliviano en estas actividades podría expresar la emergencia de nichos de trabajo protegidos que evitan los costos de emplearse en el mercado de trabajo secundario, al mismo tiempo que constituyen una vía de movilidad social ascendente para los/as migrantes (Portes y Bach, 1985).

Por otra parte, y de manera similar a las décadas previas, las formas de migrar continúan determinadas por una migración familiar en donde comúnmente el varón avanza primero. El testimonio de Julio, en tal sentido, es un buen ejemplo de lo que venimos desarrollando hasta aquí: llega solo directamente desde Bolivia hacia Córdoba a partir de la existencia de hermano viviendo previamente en la ciudad que lo llama y le consigue trabajo (en la construcción), con posterioridad arriba su esposa con sus hijos:

\section{E: Cuénteme cómo llega a Córdoba.}

J: Llego a fines de los ochenta, en el 88. La cosa estaba mal allá, no teníamos trabajo. Me vine a trabajar en la construcción, mi hermanó me llamó para que viniera, él ya estaba trabajando acá. A los 2 años vino mi mujer y mis dos hijos (Comunicación personal, Córdoba, 2005).

Elena, su esposa, relataba así su llegada a la Córdoba:

E: ¿Cuándo llega a Córdoba?

E: En el noventa, mi marido ya estaba acá.

E: ¿Por qué Córdoba?

19 Durante gran parte del siglo XX fueron los inmigrantes europeos quienes principalmente se dedicaron a la producción hortícola en fresco y a la fabricación de ladrillos, a través de emprendimientos familiares que requerían mano de obra que en un primer momento estaba conformada por trabajadores que eran migrantes internos (provenían de otras provincias argentinas) y que gradualmente fue suplantada por migrantes bolivianos. Para el paso particular de la producción de verduras y hortalizas, y como sostienen Pizarro y Trpin: "a fines del siglo XX los productores europeos y sus descendientes fueron dejando esa actividad debido, entre otras cosas, a los procesos de desplazamiento que sufrieron los productores familiares a raíz de la aplicación de políticas neoliberales y a la escasa valoración social de esa actividad considerada como un trabajo bruto, duro, sacrificado y de analfabetos. Progresivamente, algunos trabajadores bolivianos que habían logrado una movilidad socioeconómica y laboral reemplazaron a sus antiguos patrones, convirtiéndose en productores (arrendatarios o propietarios) e, incluso, en comercializadores" (2011, pp. 212-213). 
E: Yo siempre digo que nosotros nos vinimos con una valija llena de ilusiones, de proyectos para poder salir adelante, con muchas expectativas de seguir estudiando, según lo que no decían, lo que nos llamaba mucho la atención era la Universidad de Córdoba, entonces con todo eso vinimos nosotros, primero mi marido, después yo con mis hijos chiquitos. Después de 1985 en Bolivia no había trabajo. Mi esposo tiene un hermano acá, y él nos habló bonito, nos habló de lo bonito que era la ciudad, entonces llegamos a Córdoba y no nos movimos más (Comunicación personal, Córdoba, 2005).

En relación con las localizaciones territoriales, los/as migrantes bolivianos/as en Córdoba, tanto aquellos más recientes como quienes hacía un tiempo que se encontraban viviendo en la ciudad, comienzan a desplazarse hacia espacios urbanos periféricos. Si bien Villa El Libertador continúa siendo un lugar significativo -en términos cualitativos y cuantitativos- para la población boliviana en Córdoba; las condiciones socioeconómicas que enfrenta un amplio conjunto de esta población, en el marco de trayectorias laborales caracterizadas por la precariedad e informalidad, ha convertido al acceso a la vivienda en una problemática central. Así pues, obtener una vivienda, incluso un terreno, a través del mercado inmobiliario formal, ya sea propia como alquilada, resulta una posibilidad cada vez más remota (Magliano, Perissinotti y Zenklusen, 2014). De este modo, se ha producido un desplazamiento de muchos migrantes hacia zonas específicas de la ciudad, en su mayoría terrenos fiscales situados en áreas de la periferia urbana que hasta el momento de su ocupación constituían terrenos baldíos. Es entonces a partir de la apropiación de estos terrenos, la cual se mantiene en el plano informal a partir de su ilegalidad, que muchos migrantes bolivianos, especialmente quienes recién arriban, reconfiguran su presencia en el ámbito urbano cordobés.

Si bien Córdoba continúa siendo un destino elegido por muchos/as migrantes bolivianos/as ${ }^{20}$, en el transcurso del último cuarto del siglo XX fueron emergiendo nuevos destinos dentro del país en el marco del surgimiento de oportunidades laborales que posibilitaban, según los testimonios de los propios migrantes, ahorrar dinero y resolver ciertas problemáticas presentes en las grandes ciudades del país (el acceso a la vivienda es uno de ellos). La llegada a la ciudad de Ushuaia, en el extremo sur del país, puede considerarse como un nuevo escenario en la continuidad de esta segunda edad de migrantes bolivianos/as y, a la vez, como la expresión de un tercer momento en la historia de la migración boliviana en Argentina que, a la vez que continúa llegando a los grandes centros urbanos, incorpora otros destinos en pos de un proyecto laboral.

20 De acuerdo a los datos del censo nacional de población del 2010, Córdoba se transformó en la provincia en la que el crecimiento de la población nacida en otro país, en especial boliviana y peruana, fue mayor: el 42,8\% del total llegó entre 2002 y 2010 (INDEC, 2012). 


\section{La tercera edad de la migración boliviana en Argentina: el sur también existe}

La presencia de bolivianos/as en la Patagonia Austral comienza a visibilizarse durante los años $80^{21}$. Para el caso de Ushuaia, los primeros bolivianos que arriban dando forma a la actual presencia boliviana en la ciudad son varones trabajadores de la construcción, residentes (es decir migrantes) en otras provincias argentinas, entre ellas Córdoba. Los trabajadores bolivianos llegan contratados por empresas constructoras para trabajar en la ciudad, especialmente en grandes obras públicas que expresan una ciudad en transformación ${ }^{22}$. Asimismo, la gran mayoría llega a Argentina en el marco de la segunda edad de la migración, es decir en la segunda mitad del siglo XX, y el arribo a Ushuaia es producto de la movilidad característica de este tipo de trayectorias y de los efectos de las dinámicas poblacionales internas dentro de Argentina.

Sin embargo, existen algunas diferencias importantes que permiten comprender la gestación de un nuevo momento en la tradición migratoria boliviana en el país. Primero, el papel del Estado. Para el caso de Ushuaia, los flujos son producto de un esfuerzo por parte del Estado argentino para poblar territorios definidos como vacios. Los diferentes incentivos para la relocalización de la población argentina tienen su impacto en la población boliviana residente en el país que decide su traslado y, a veces, su residencia en la ciudad. Al igual que algunas de las dinámicas comprendidas en la segunda edad, nos referimos a la migración interna de migrantes bolivianos; este movimiento no se incluye en una nueva corriente migratoria hacia Argentina sino que, por el contrario, forma parte de los flujos migratorios (internos) que provienen de múltiples localidades argentinas hacia la ciudad del sur. Es decir que los ya migrantes utilizan tanto como los nativos los recursos e incentivos otorgados por los estados. Esta relación con el Estado en tanto ciudadano/a-residente (y ya no como migrante) será, como veremos más adelante, uno de los rasgos centrales que caracterizan la migración boliviana hacia Ushuaia y que incluimos en esta tercera edad. Se trata de una relación con el Estado que no se asocia a la condición de migración (los movimientos de los/as bolivianos/as como en otras etapas no pueden ser pensados como una política poblacional por parte del estado) sino a una

21 En consonancia con la llegada de migración boliviana a la región sur del país surgieron trabajos que analizan esa presencia, entre ellos, Baeza (2011 y 2013), Radonich, Ciarallo y Trpin (2013).

22 En 1972, el gobierno nacional sanciona un régimen de promoción económica basado en exenciones arancelarias e impositivas: la Ley 19.640 -ratificada en 1974 por el Congreso Nacional- que pretendía (y lo logró) estimular la radicación de industrias. Éstas se vieron atraídas por la posibilidad de elaborar artículos en Tierra del Fuego a partir de materias primas importadas, y la posibilidad de exportar esos productos al territorio continental nacional sin que su primera venta fuera gravada con el impuesto al valor agregado (IVA). 
relación expresadas en ciertas demandas que se realizan en tanto residentes/habitantes (independientemente de la condición de nacionalidad). En el caso de Ushuaia, los incentivos generados desde el Estado para fomentar la residencia de los/as trabajadores temporales serán también parte de los argumentos esgrimidos por las primeras familias bolivianas para quedarse en la ciudad cuya llegada a la ciudad se comprende más como el efecto de las facilidades otorgadas a las empresas durante los años 70 y 80 . Así recuerdan Meri y Óscar el arribo a Ushuaia:

Ó: A Ushuaia llegué por una empresa, en Córdoba. Era subsidiaria de otra. Era una contratista. Yo vine porque me pagaban un buen sueldo, como todos [...] O sea que vine bien. El contrato era por un año, con opción a estirarse.

E: ¿Y dónde vivía?

Ó: Estaba viviendo en un alojamiento que nos alquilaron de la misma empresa. En una gamela pero era para maquinistas (Óscar, comunicación personal. Ushuaia, 2008).

M: Y después viene otro amigo del barrio también ahí que cayó a Bahía Blanca, ahí a la empresa, y trabajaba con nosotros y él dice: "Me voy para Ushuaia", así, así. "Me voy a trabajar un par de meses porque mi mamá se fue" [...] Como siempre, entre los conocidos amigos nos pasamos el dato: tal empresa está tomando gente para tal lugar. Así formamos un grupo y vamos un grupo. Entonces ahí un paisano me dice: "Mira, tal empresa toma peones para Ushuaia", y yo fui (Meri, comunicación personal. Ushuaia, 2006).

Y Ema, en relación con la llegada de su marido:

E: ¿¿sted en qué año llegó a Ushuaia?

E: En el 81. Mi marido vino primero porque nosotros conocíamos una empresa que lo traía, por eso vino a trabajar, pensando que le va a ir bien (Comunicación personal, Ushuaia, 2006).

A partir de las entrevistas realizadas, podemos decir que en líneas generales los pioneros llegan a la ciudad de manera individual en un tipo de proyecto familiar donde primero migran los varones y después las mujeres. En conjunto son proyectos de relocalización familiar vinculados con la perspectiva de progreso comprendidos no sólo en 
términos económicos sino de acceso a la educación, salud y, sobre todo, a una vivienda propia. Se trata de personas de mediana edad, ya unidas y radicadas en Argentina, y que en una ciudad que no eligieron encuentran oportunidades estructurales que las motivan a volver o a quedarse. En este caso, fueron políticas estatales -económicas y poblacionales- las que explícitamente fomentaron la llegada de las empresas y de las personas que serían empleadas como mano de obra, entre ellos algunos varones bolivianos trabajadores de dichas empresas. A partir de políticas empresariales se organizan las llegadas y se decide el lugar y el período de trabajo. Así, el viaje no es efecto inmediato de la voluntad de moverse al lugar de destino, sino que está determinado por el lazo de unión con la empresa que ofrece este tipo de estadías en diferentes lugares del país, compatibles con la posibilidad de acumular y ahorrar dinero (los costos de hospedaje y comida en las jornadas laborales están cubiertos por las propias empresas). En otras palabras, es la relación con la empresa lo que da sentido al desplazamiento.

En sus inicios, si la necesidad de mano de obra funcionó como uno de los elementos de atracción hacia la ciudad de Ushuaia, con posterioridad las redes migratorias organizaron la llegada de obreros de la construcción bolivianos (junto con argentinos y chilenos) provenientes de diferentes lugares del país, primero como empleados de constructoras y luego como parte de proyectos migratorios encabezados por los varones.

Aquellos que primero arribaron se establecen como trabajadores temporarios, habitando en pensiones o piezas rentadas, y retornando después de cierto plazo a sus residencias habituales en otras provincias de la Argentina. Sólo algunos de ellos deciden permanecer, convirtiéndose en los primeros pobladores bolivianos de Ushuaia. En la mayor parte de los casos se trata de varones con residencias estables en alguna de las grandes localidades argentinas (especialmente Buenos Aires y Córdoba), ya unidos y generalmente con hijas/os. Por lo tanto, en sus relatos, el transformarse en migrantes fueguinos y la elección por la permanencia toma sentido con la llegada de sus mujeres y familias: el pasaje de lo temporario a proyectos de larga duración es relatado en términos conyugales.

El atractivo para ellos no se agota en el diferencial económico de los sueldos recibidos, sino sobre todo en la posibilidad de contar con recursos estatales incluidos en políticas destinadas a fomentar la instalación de las/os trabajadoras/es en la ciudad, como el acceso a planes de vivienda y amplios beneficios sociales. Esto supone migrantes regularizados (condición para acceder a los beneficios), cuyos movimientos se vinculan con estrategias de ascenso social (por ejemplo, llegar a ser propietarias/os) y con intenciones de permanecer (al menos un tiempo) en una ciudad argentina. 
M: Y empezamos a trabajar y nos quedamos tres años y aquí nació mi primer hijo.

E: Un montón. ¿Y querían ustedes quedarse?

M: Y bueno, como dio trabajo, habíamos venido de allá en busca de trabajo, de progresar económicamente. Por eso, bueno, se nos presentó el trabajo y trabajamos (Meri, comunicación personal. Ushuaia, 2006).

G: Nosotros nos quedamos porque ya estamos medianamente establecidos... Tenemos casa, trabajo, los chicos van a la escuela... siguen pasando los años y seguimos armando proyectos (Germán, comunicación personal. Ushuaia, 2005).

A: ¿Y tenía ganas de quedarse usted acá?

J: Bueno, ahora mismo no puedo terminar de preguntarme por qué me quedaba. La gente venía por temporarios nada más; después trabajaban unos seis meses y se iban y así yo me quedaba porque nomás yo tenía ya la casita toda y mis hijos y bueno, así me quedé (Juana, comunicación personal. Ushuaia, 2006).

El resto de las mujeres y los varones bolivianos arriban a la ciudad por efecto de la activación de redes migratorias, a partir de la relación (más o menos mediada) con personas ya radicadas en la ciudad. Las redes migratorias hacia Ushuaia se inician con el retorno a sus ciudades de residencia o a otros destinos laborales de los primeros trabajadores, pioneros o no, contratados por las empresas constructoras y continúan iniciándose aún en la propia Bolivia.

De acuerdo a lo que hemos podido reconstruir, las condiciones favorables para la permanencia otorgadas por el Estado local para fomentar la radicación definitiva de los contingentes migratorios (especialmente el acceso a la vivienda ${ }^{23}$ ) se visibilizan como una oportunidad no vivida en otras provincias argentinas, como mencionamos para el caso de Córdoba. Ushuaia, en este sentido, permite la posibilidad de pensar residencias sedentarias (al menos en el mediano plazo) que son la vía de acceso a derechos y programas de gobierno (el más mencionado es el acceso a la vivienda vía el Instituto Provincial de la

23 El acceso a la vivienda vía los programas del Instituto Provincial de la Vivienda IPV son el modo predominante en que las primeras familias bolivianas acceden a su casa propia en la década del 80. Esta posibilidad funciona luego como expectativa realizable para los/as bolivianos/as que llegan con posteridad aunque el acceso con los años se haya vuelto más dificultoso. En la actualidad y desde hace algunos, Ushuaia se encuentra en crisis habitacional. 
Vivienda IPV) ofrecidos por el Estado. Así, es en Ushuaia que nuestros/as entrevistados/ as se convierten en "migrantes" en tanto aspiran a las formas de ciudadanías ofrecidas por el Estado de la sociedad receptora.

Pero también Ushuaia canaliza otras virtudes: trabajos menos sacrificados, según cuentan quienes residieron en Buenos Aires, Mendoza o Córdoba, donde se trabaja en la producción y comercialización de hortalizas de sol a sol, o en talleres textiles donde el jornal se calcula a destajo; en fin, todas actividades que requieren un enorme sacrificio físico en el desplazamiento y circulación por la ciudad, que disminuye en Ushuaia, especialmente para las mujeres. En palabras de Gladys:

G: Cuando me fui de acá de dos años, me fui, extrañando a mi mamá a verlo a mi mamá y cuando llegué dije 'nunca más no voy a volver a este Ushuaia' Después cuando me fui a mi casa y no me quería quedarme, quería venirme de vuelta acá.

E: ¿Y por qué?

G: No sé. Ya no estaba acostumbrada a trabajar, como que era fuerte el trabajo para mí.

E: ¿es muy fuerte el trabajo allá?

G: Con el pico, con la pala, tener que cavar, todas las verduras, todo tenés que hacer, todo el día y domingo tampoco no descansas. Todo el día, toda la semana que trabajar. (Comunicación personal, Ushuaia, 2006)

A partir del trabajo de campo realizado en Ushuaia, podemos inferir que el tiempo de permanencia de la población boliviana en las ciudades argentinas, el desarrollo de redes familiares y de paisanazgo y la especialización del trabajo en ciertos nichos, entre otros factores, ha permitido que un sector experimente trayectorias ascendentes en términos socio-económicos, haciendo propio los beneficios de poblar la Patagonia. En pocos años acceden a la vivienda, dentro de la construcción se organizan como contratistas (con grupos de trabajo exclusivamente de origen boliviano), las mujeres se retiran del mercado de trabajo o realizan tareas que son consideradas livianas (vinculadas a los servicios por fuera de los espacios domésticos) y quienes tienen hijos/as se escolarizan con aspiraciones universitarias. 
Las llegadas a Ushuaia muestran que son las características de la segunda edad las que permiten la inflexión positiva en las trayectorias económicas de algunas familias bolivianas que ascienden económica y socialmente y que generan nuevas redes migratorias para proveerse de mano de obra familiar y boliviana (donde el lazo de confianza es primordial en el tipo de trabajo informal en el que se desempeñan).

Un elemento adicional de las llegadas y permanencias en el sur es el deseo de convertirse en ciudadanos/as de los lugares ( $\mathrm{y}$ a veces de los estados) de residencia. Ushuaia nos muestra esta transformación: algunos/as residentes se posicionan como ciudadanos frente al Estado, reclamando y demandando los mismos derechos que los residentes nacionales. La justificación generalmente se nutre de su inserción en el mercado, la inversión monetaria realizada en la ciudad y el pago de impuestos que en su visión los interpela como ciudadanos/as. Se trata de una ciudadanía reclamada que supera ampliamente lo formal (el hecho de obtener residencias regulares en Argentina) y que apunta a una ciudadanía práctica enmarcada en la posibilidad de gozar de derechos: a la salud, educación, vivienda, etc. En las entrevistas, los/as bolivianos/as, integrados/as en el mercado laboral, generadores de ingresos y tributarios del Estado argentino demandan para sí los mismos derechos que los/as nacionales a partir de los discursos de igualdad formal contenidos en las nociones de ciudadanía formal del mundo moderno occidental. En Ushuaia, esto se ha expresado especialmente en demandas por el derecho a la vivienda. De esta manera, esta edad de la migración boliviana es fruto de la permanencia en el país, siendo la sedentariedad vivida como expresión misma del ascenso social que habilita a visualizarse como residentes-ciudadanos/as sin importar el origen extranjero.

De modo similar a lo ocurrido en otras ciudades -especialmente en Buenos Aires de acuerdo a los trabajos de Canelo (2013) y Gallinati (2008)-, migrantes bolivianos se organizan no sólo en términos de reivindicaciones comunitarias o culturales sino como habitantes o vecinos en el reclamo de derechos generando posibilidades de articulación política con los locales (aún poco elaboradas en el caso de la ciudad de Ushuaia). Permanencias prolongadas (propias o de la colectividad), residencias estables y una particular vinculación con el Estado posibilitan estas dinámicas que, a modo de hipótesis, proponemos como rasgo diferencial de una tercera edad de la migración. 


\section{Conclusión}

Las edades migratorias aquí reconstruidas como una excusa para reflexionar sobre las especificidades socio-históricas de la migración boliviana hacia Córdoba y Ushuaia y las distintas generaciones que involucra este proceso en Argentina, expresan diferentes temporalidades, formas de llegada y condiciones de posibilidad articuladas con ciertos procesos históricos y sociales tanto del país de origen como de destino. Esto explica el dinamismo histórico de la migración boliviana, las diversas localizaciones territoriales y sus trayectorias socio-laborales y políticas.

Analizando en clave comparativa los casos de Córdoba y Ushuaia, en particular, es posible percibir que ambas localidades expresan diferentes momentos en la larga historia que tiene la migración boliviana en Argentina. Córdoba tiene una mayor antigüedad en la recepción de personas bolivianas que además presentan trayectorias migratorias y laborales más heterogéneas a partir de la pertenencia de clase y el origen étnico-nacional. Ushuaia puede considerarse como un espacio novedoso en la instalación de migrantes de origen boliviano en el que las trayectorias de los pioneros signan las características de la población: masculinizada y perteneciente a la clase trabajadora (especialmente asociados a la industria de la construcción). El hecho de recuperar las especificidades de ambos espacios ha permitido también identificar ciertas particularidades de los mercados de trabajo locales y del rol de Estado. Entre los aspectos en común, aparece la centralidad de la migración familiar y laboral. Aunque los varones hayan sido en muchos casos los pioneros de la migración, la decisión de migrar no se constituye como una decisión individual sino que, por el contrario, involucra a toda la familia. En tal sentido, la migración de la mujer, generalmente asociada a la de los/as hijos/as, ha sido un factor central para la estabilidad del proyecto migratorio, especialmente en términos de sedentariedad.

El peso de las redes sociales, por su parte, ha resultado clave a la hora de leer y reconstruir las edades de la migración boliviana. La larga tradición migratoria boliviana en Argentina, siempre presente como estrategia familiar, ha permitido la constitución de los movimientos como una estructura permanente entre ambos países. Cada nueva generación de migrantes llega al país y encuentra ya establecida una comunidad de emigrantes más antiguos, a la cual puede agregarse (Mallimaci, 2012, p. 181). En tal sentido, tanto en Córdoba como en Ushuaia, varones o mujeres, no emigran de modo autónomo, si por ello se entiende soledad o independencia de estructuras relacionales. En los testimonios, la elección por Argentina es sostenida por el lazo histórico entre ambos países, conformado por las constantes movilidades provenientes de Bolivia. La vasta, difundida e histórica experiencia migratoria queda expresada en el conocimiento directo y com- 
partido por todos/as los/as entrevistados/as sobre historias de vida de personas cercanas (vecinos/as, familiares) que han viajado y viajan a la Argentina: desde siempre han sido socializados/as con imágenes de personas que parten hacia Argentina, vuelven y, a veces, parten nuevamente.

\section{Referencias}

Baby-Collin, V. y Cortes, G. (2014). Nuevos despliegues del campo migratorio boliviano frente a la crisis. Revista CIDOB d'Afers Internacionals, 106-107, 61-83.

Baeza, B. (2011). Migración boliviana en Comodoro Rivadavia (Chubut): asociacionismo y lazos transnacionales. En C. Pizarro (Coord.), Migraciones internacionales contemporáneas. Estudios para el debate (pp. 223-243). Buenos Aires, Argentina: CICCUS.

- - (2013). Trabajadores de la construcción: bolivianos y paraguayos en Comodoro Rivadavia. Identificaciones, diferenciaciones y conflictos. En G. Karasik (Coord.), Migraciones internacionales. Reflexiones y estudios sobre la movilidad territorial contemporánea (pp. 361-382). Buenos Aires, Argentina: CICCUS.

Balan, J. (1985). Las migraciones internacionales en el Cono Sur. Buenos Aires, Argentina: Cedes.

- - - (1990). La economía doméstica y las diferencias entre los sexos en las migraciones internacionales: un estudio sobre el caso de los bolivianos en Argentina. Estudios Migratorios Latinoamericanos, 15-16, 269-294.

Bendini, M. y Radonich, M. (1999). De golondrinas y otros migrantes. Buenos Aires, Argentina: La Colmena.

Benencia, R. (1997). De peones a patrones quinteros. Movilidad social de familias bolivianas en la periferia bonaerense. Estudios Migratorios Latinoamericanos, 35, 63-102.

- - . (2003). Apéndice: La inmigración limítrofe. En F. Devoto, Historia de la inmigración en Argentina (pp. 433-484). Buenos Aires, Argentina: Editorial Sudamericana.

- - - (2012). Participación de los inmigrantes bolivianos en espacios específicos de la producción hortícola argentina. Revista Política y Sociedad, 49 (1), 163-178. 
Benencia, R. y Karasik, G. (1995). Inmigración limítrofe: los bolivianos en Buenos Aires. Buenos Aires, Argentina: CEAL.

Bologna, E. (2007). Jujuy: movilidad ancestral, tierra de paso y vínculos estables. En H. Domenach; D. Celton; H. Arze y P. Hamelin (Eds.), Movilidad y procesos migratorios en el espacio de frontera Argentino-Boliviana (pp. 175-218). Córdoba, Argentina: Centro de Estudios Avanzados, Universidad Nacional de Córdoba.

Bompadre, J. M. (2005). La fiesta como espacio de discurso y prácticas sociales: El caso de la Virgen de Urkupiña en Córdoba. En E. Domenech (Comp.), Migraciones contemporáneas y diversidad cultural en la Argentina (pp. 203-224). Córdoba, Argentina: Centro de Estudios Avanzados.

Bourdieu, P. (2011). Las estrategias de la reproducción social. Buenos Aires, Argentina: Siglo XXI Editores.

Canelo, B. (2013). Fronteras internas: migración y disputas espaciales en la Ciudad de Buenos Aires. Buenos Aires, Argentina: Antropofagia.

Ceriani, P., Courtis, C., Pacecca, M. I., Asa, P. y Pautassi, L. (2009). Migración y trabajo doméstico en Argentina: las precariedades en el marco global. En M. E. Valenzuela y C. Mora (Eds.), Trabajo doméstico: un largo camino hacia el trabajo decente (pp. 147-190). Santiago de Chile: OIT.

Cortés, G. (2004). Una ruralidad de la ausencia. Dinámicas migratorias internacionales en los valles interandinos de Bolivia en un contexto de crisis. En A. Hinojosa (Comp.), Migraciones transnacionales. Visiones de Norte y Sudamérica (pp. 167-199). La Paz, Bolivia: Plural Editores.

Dandler, J. y Medeiros, C. (1991). Migración temporaria de Cochabamba, Bolivia, a la Argentina: Patrones e impacto en las áreas de envío. En P. Pessar (Comp.), Fronteras permeables (pp. 19-54). Buenos Aires, Argentina: Planeta.

De la Torre Ávila, L. (2006). No llores, prenda, pronto volveré. Migración, movilidad social, herida familiar y desarrollo. La Paz, Bolivia: PIEB/IFEA/UCB.

Devoto, F. (2004). Las políticas migratorias de Francia y Argentina en el largo plazo. Estudios Migratorios Latinoamericanos, 53, 121-153. 
Domenach, H. y Celton, D. (1998). La comunidad boliviana en Córdoba. Caracterización y proceso migratorio. Córdoba, Argentina: ORSTOM, Universidad Nacional de Córdoba.

Farah, I. (2005). Migraciones en Bolivia: estudios y tendencias. Umbrales, 13, 135-168.

Ferrufino, C. (2007). Los costos humanos de la emigración. Cochabamba, Bolivia: Universidad Mayor de San Simón, PIEB.

Gallinati, C. (2008). ¿Inmigrantes o ciudadanos? La construcción del 'verdadero' modelo de política migratoria en el marco de la 'patria grande'. Publicación del CIFFyH, 5, 479500.

Hinojosa, A. (2004). Migraciones transnacionales. Visiones de Norte y Sudamérica. La Paz, Bolivia: CEF/Plural.

-_- (2009). Buscando la vida. Familias bolivianas transnacionales en España. La Paz: CLACSO.

Instituto Nacional de Estadística y Censo-INDEC (2012). Censo nacional de población, hogares y viviendas 2010: censo del Bicentenario: resultados definitivos. Buenos Aires, Agentina: Instituto Nacional de Estadística y Censos (INDEC).

Karasik, G. (2011). Sobre-etnización y epistemologías de extranjerización. Reflexiones a partir del caso de Jujuy como contexto de migraciones bolivianas (tempranas) hacia Argentina. En C. Pizarro (Coord.), Migraciones internacionales contemporáneas. Estudios para el debate (pp. 231-256). Buenos Aires, Argentina: CICCUS.

López, V. (1999). Migración limitrofe en la ciudad de Córdoba. Córdoba: Serie de Estudios de Población, Maestría en Demografía, Facultad de Ciencias Económicas, Universidad Nacional de Córdoba.

Magliano, M. J. (2013). Los significados de vivir 'múltiples presencias': Mujeres bolivianas en Argentina. Migraciones Internacionales, 7 (24), 165-195.

Magliano, M.J., Perissinotti, V. y Zenklusen, D. (2014). Estrategias en torno a las formas de apropiación y organización del espacio en un 'barrio de migrantes' de la ciudad de Córdoba. Revista Estudios Demográficos y Urbanos, 29 (3), 513-539. 
Mallimaci, A. I. (2012). Moviéndose por Argentina: Sobre la presencia de bolivianos en Ushuaia. Migraciones Internacionales, 6 (23), 173-207.

Marshall, A. y Orlansky, D. (1983). Inmigración de países limítrofes y demanda de mano de obra en la Argentina, 1940-1960. Desarrollo Económico, 89 (23), 34-48.

Pizarro, C. y Trpin, V. (2011). Trabajadores frutícolas y hortícolas en la Argentina. Una aproximación socioantropológica a las prácticas de reproducción y de resistencia de las condiciones laborales. Revista Ruris, 4 (2), 199-228.

Portes, A. y Bach, R. (1985). Latin Journey. Cuban and Mexican Immigrants in the United States. Berkeley, Estados Unidos: University of California Press.

Pries, L. (2002). Migración transnacional y la perforación de los contenedores de Estadosnación. Revista Estudios Demográficos y de Desarrollo Urbano, 51, 571-579.

Radonich, M., Ciarallo, A. y Trpin, V. (2013). Chilenos y bolivianos en la conformación de territorios en áreas rurales del Alto Valle de Río Negro, Argentina. En G. Karasik (Coord.), Migraciones internacionales. Reflexiones y estudios sobre la movilidad territorial contemporánea (pp. 379-400). Buenos Aires, Argentina: CICCUS.

Reboratti, C. (1983). Peón golondrina: cosechas y migraciones en la Argentina. Cuaderno del Cenep, 24, 1-36.

Rivero Sierra, F. (2012). Pagos chicos y pagos grandes, articulación entre migraciones internas e internacionales. El caso de los campesinos potosinos de Toropalca. Estudios Migratorios Latinoamericanos, 26, 75-102.

Sassone, S. (1988). Migraciones laborales y cambio tecnológico. El caso de los bolivianos en el Ramal Jujeño. Cuadernos de Antropología Social, 1, 97-112.

- - - (2009). Breve geografía histórica de la migración boliviana hacia Argentina. En Comisión para la Preservación del Patrimonio Histórico Cultural, Buenos Aires boliviana. Migración, construcciones identitarias y memoria (pp. 389-402). Buenos Aires, Argentina: Comisión para la Preservación del Patrimonio Histórico Cultural. 
Sayad, A. (1977). Les trois âges' de l'émigration algérienne en France. Actes de la recherche en sciences sociales, $15,59-79$.

Tarrius, A. (2002). La mondialisation par le bas. Les nouveaux nomades de l'économie souterraine. Paris, Francia: Balland.

Zalles, A. (2002). El enjambramiento cultural de los bolivianos en Argentina. Nueva Sociedad, 178, 89-103. 